\title{
Alcohol intake and survival in Australian seniors: The Dubbo Study
}

\author{
L.A. Simons* \\ School of Medicine, University of NSW, Kensington, NSW, Australia
}

\begin{abstract}
Consumption of moderate amounts of alcohol, compared with abstention or with heavy intake, appears to be associated with reduced all-cause mortality (ACM) in middle-aged subjects. The Dubbo Study of Australian elderly is a longitudinal study of healthy ageing. In 1988-89 we examined 2805 non-institutionalised citizens 60+ years of age born before 1930, mean age 69 years. The cohort comprised 1233 men and 1572 women, representing 73\% of the eligible population. This report examines the relationship between alcohol intake and mortality in this cohort during follow-up over 20 years. Alcohol intake was arbitrarily grouped into 4 categories: nil, low, moderate and heavy. The analysis could not distinguish between intake of wine versus intake of spirits. $78 \%$ of men and 52\% of women reported some alcohol intake. Most men reported an intake of 1-14 drinks/week, most women 1-7 drinks/week; $87 \%$ of male drinkers and $44 \%$ of female drinkers predominantly consumed beer and the remainder consumed wine and/or spirits. Over 20 years to $2008,66 \%$ of men and $53 \%$ of women died; $64 \%$ of male drinkers died versus $72 \%$ of non-drinkers; $46 \%$ versus $60 \%$ in females. In a multivariate model, ACM was related to quantity of alcohol intake in the familiar "U" shaped relationship, being $20 \%$ and $28 \%$ reduced in the low and moderate intake categories respectively, compared with nil intake. This relationship was similar in men and women, and with intake of beer or wine/spirits. Any alcohol intake added 12 months survival time in men and women over the follow-up period. Alcohol intake in the low to moderate range appeared to offer protection against the onset of dementia. The overall Dubbo results are not unique, but are applicable to senior citizens. A "healthy survivor" effect may be partially operating in this context.
\end{abstract}

Keywords: Alcohol intake, elderly, all-cause mortality, dementia

\section{Introduction}

Consumption of moderate amounts of alcohol, compared with abstention or with heavy intake, appears to be associated with reduced all-cause mortality (ACM) in middle-aged subjects [1-4]. This effect may be partially mediated through a reduced risk of cardiovascular disease [5-7]. Some studies attribute the reduced ACM to a specific effect of wine [8, 9], other studies attribute it to any type of alcohol [5]. In elderly people some of this benefit from moderate alcohol intake may be negated by mortality from other causes $[4,10]$.

The Dubbo Study of Australian elderly is a longitudinal study of healthy ageing [11, 12]. In 198889 we examined 2805 non-institutionalised citizens

\footnotetext{
*Corresponding author: L.A. Simons, School of Medicine, University of NSW, Kensington, NSW 2033, Australia. E-mail: L.Simons@unsw.edu.au.
}

$60+$ years of age born before 1930 , mean age 69 years. The cohort comprised 1233 men and 1572 women, representing $73 \%$ of the eligible population in the Local Government Area of Dubbo, NSW. About $66 \%$ of men and $53 \%$ of women died during 20 years follow-up [12].

We have previously reported on the relationship between alcohol intake and mortality in this cohort during follow-up of about 10 years [13]. In the present report we have examined this relationship during follow-up extended to 20 years.

\section{Methods}

Methods and measures have been described in detail previously [11]. Briefly, the baseline examination comprised standard demographic, psychosocial 
and cardiovascular assessments, including examination of fasting blood samples. The study population were broadly representative of the Australian population born before 1930 by gender, age, employment, socioeconomic status, housing tenure, mean blood pressure and other variables.

Questions on alcohol use were identical to those asked in the National Heart Foundation Risk Prevalence Study [22], and yielded an approximation of usual alcohol intake coded as zero, 1-7, 8-14, 15-28 and $>28$ drinks/week (referring to a standard drink containing $10 \mathrm{~g}$ of alcohol). Specific intakes of beer, wine and spirits were not separately sought, but subjects were asked if they normally drank beer or not, allowing a separation of drinking behaviour into beer and "other" (i.e. other is the combined group of wine and/or spirits).

From the weekly intakes listed above, we have arbitrarily grouped alcohol intake into four categories - nil, low, moderate and heavy. The respective sex-specific definitions were as follows:

- Low: men 1-14 drinks/week, women 1-7 drinks/week

- Moderate: men 15-24 drinks/week, women 8-14 drinks/week

- Heavy: men 25+drinks/week, women $15+$ drinks/week

Deaths were ascertained from hospital or public records, with postal surveys conducted every two years to confirm vital status. The independent contributions of potential predictors and confounders to ACM were examined in Cox proportional hazards models. Point estimates and 95\% confidence intervals for the risk of ACM were calculated from the regression coefficients (presented as a hazard ratio, a measure of relative risk). The Dubbo Study has received approval from institutional ethics committees at St Vincent's Hospital Sydney, the University of NSW and the University of Western Sydney, and all subjects gave informed, written consent.

\section{Results}

The pattern of alcohol intake is shown in Fig. 1. Almost 4 in 5 men and 1 in 2 women took some alcohol. There were more women in the moderate versus heavy intake categories. The heavy intake category was relatively small in both sexes. Men predomi-

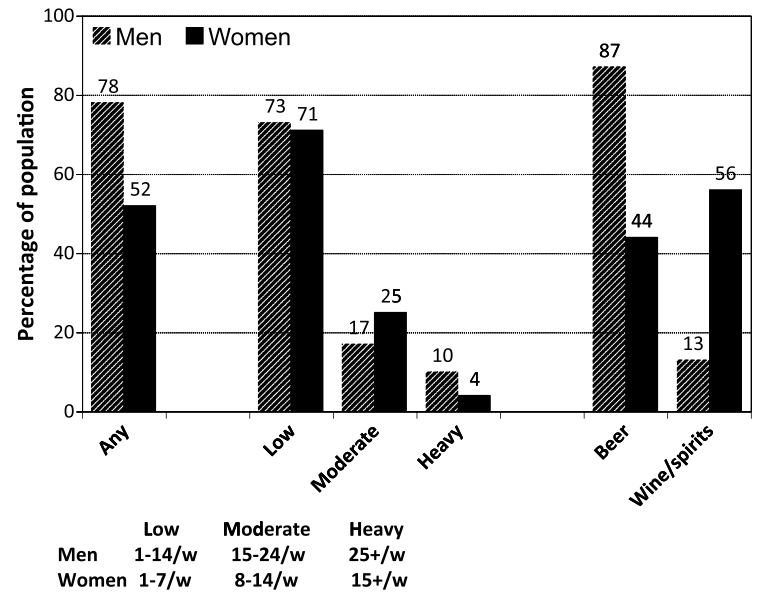

Fig. 1. Pattern of alcohol intake.

nantly consumed beer while women reported a mixed intake.

Over 14 years from 1988, various community outcomes were compared in 1779 subjects who took some alcohol versus 1011 subjects who abstained. Death occurred in $41 \%$ versus $47 \%$; any hospitalisation in $94 \%$ versus $93 \%$; admission to a low dependency hostel in $4.7 \%$ versus $8.9 \%$; admission to a nursing home in $6.5 \%$ versus $12.6 \%$. By the 20 year follow-up in 2008 , male deaths had occurred in $64 \%$ versus $72 \%$; female deaths in $46 \%$ versus $60 \%$. These are univariate statistics and multivariate modelling is required to see genuine relationships.

\section{Multivariate models of ACM}

The hazard ratios for ACM by alcohol intake in Cox models controlling for age (and sex in "all subjects" model) are presented in Table 1. In men and in women there was a lower risk of ACM at low and moderate alcohol intake compared with nil or heavy intake, the familiar "U-shaped" relationship. Statistical power in the heavy intake category was much reduced due to smaller subject numbers.

Given the similar relationships apparent here in men and women, and in the interest of increasing statistical power, subsequent models combined the sexes. Table 1 clearly demonstrates that all subjects in the low and moderate intake categories have a significant $25 \%$ lower risk of ACM compared with other categories.

The hazard ratios for ACM by type of alcohol intake in Cox models controlling for age, sex, and a list of 
Table 1

Hazard ratios and $95 \%$ confidence intervals for all-cause mortality by alcohol intake in Cox models controlling for age (and sex in "all subjects" model)

\begin{tabular}{lcccc}
\hline & Nil & Low & Moderate & Heavy \\
\hline Men & 1.00 & $0.82(0.69-0.97)$ & $0.88(0.69-1.12)$ & $1.08(0.81-1.44)$ \\
Women & 1.00 & $0.73(0.62-0.85)$ & $0.66(0.52-0.82)$ & $1.10(0.74-1.65)$ \\
All subjects & 1.00 & $0.76(0.68-0.85)$ & $0.75(0.64-0.89)$ & $1.06(0.84-1.33)$ \\
\hline
\end{tabular}

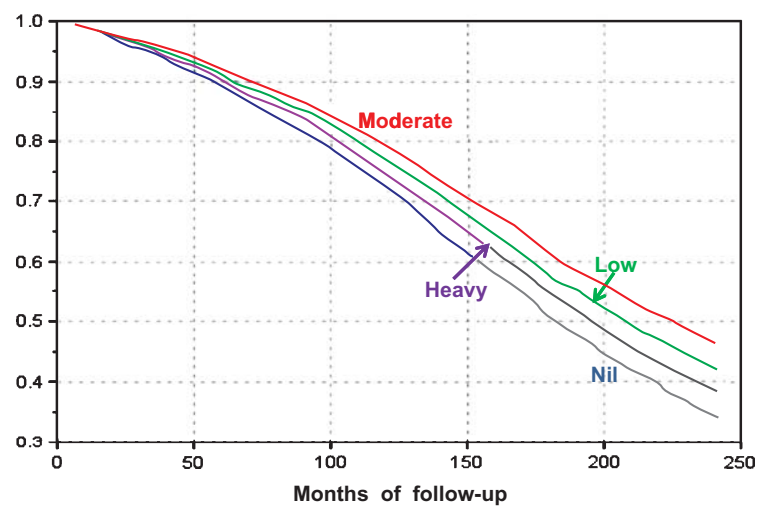

Fig. 2. Survival function for all-cause mortality by category of alcohol intake in full Cox model - all subjects.

other specified variables are presented in Table 2. For all subjects taking any type of alcohol, ACM was significantly reduced in the low and moderate categories (by $20 \%$ and $28 \%$ respectively versus nil intake). There was a broadly similar reduction in ACM whether the predominant intake was beer or wine/spirits. The hazard ratio for heavy intake of wine/spirits had very wide confidence intervals, but the point estimate did suggest a trend towards excess ACM.

The differential findings for intake categories in Table 2 are shown in Figs. 2, 3 and 4 which are the respective survival functions in the full Cox models by intake category for all alcohol use, beer only and wine/spirit only. The potentially poorer survival in the wine/spirit drinkers belonging to the heavy intake category is again apparent in Fig. 4.

\section{Potential mediators or confounders}

We attempted to define the contribution of potential mediators or confounders, such as diabetes, hypertension, body mass index or HDL cholesterol, to the relationships shown in Table 2 . We started with a

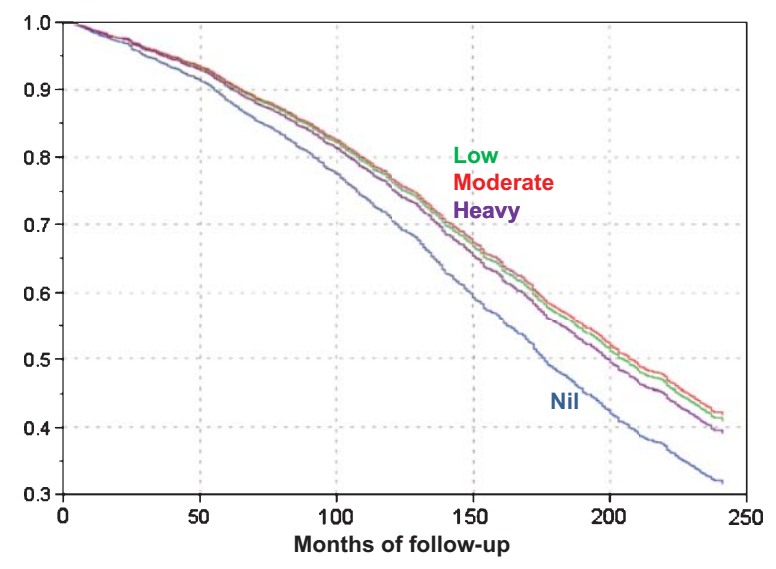

Fig. 3. Survival function for all-cause mortality by category of alcohol intake in full Cox model - beer only.

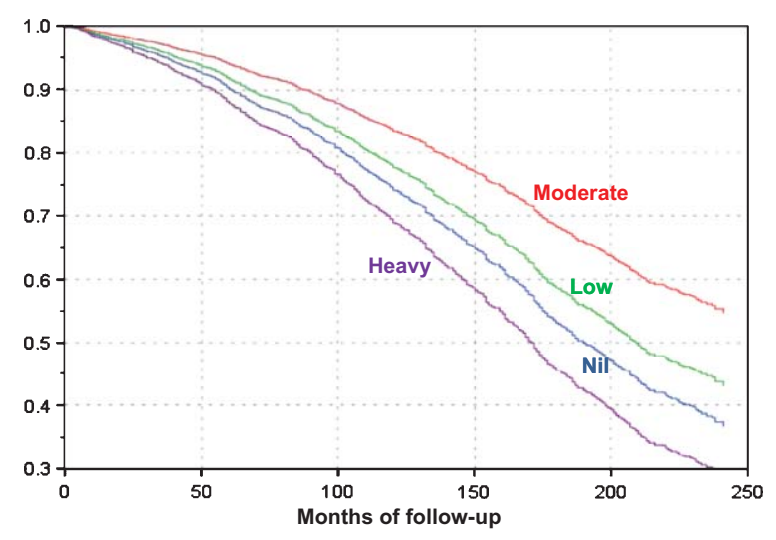

Fig. 4. Survival function for all-cause mortality by category of alcohol intake in full Cox model - wine/spirits only.

"base" Cox model which initially omitted these four variables. They were then added one at a time in a stepwise fashion. The findings are summarised in Table 3. In brief, the relationship between category of alcohol intake and ACM was not influenced by the stepwise addition of each of these variables. 
Table 2

Hazard ratios and 95\% confidence intervals for all-cause mortality by alcohol intake and type in Cox models controlling for age, sex and other predictors or potential confounders listed below

\begin{tabular}{lcccc}
\hline & Nil & Low & Moderate & Heavy \\
\hline All types & 1.00 & $0.80(0.72-0.90)$ & $0.72(0.61-0.85)$ & $0.89(0.70-1.13)$ \\
Beer & 1.00 & $0.77(0.68-0.89)$ & $0.77(0.64-0.95)$ & $0.84(0.64-1.11)$ \\
Wine/spirits & 1.00 & $0.85(0.73-1.00)$ & $0.62(0.46-0.85)$ & $1.29(0.81-2.08)$ \\
\hline
\end{tabular}

Variables in models include: age, sex, marital status, smoking, body mass index, diabetes, prior cardiovascular disease, hypertension, atrial fibrillation, peak expiratory flow, self-rated health and physical disability.

Table 3

Hazard ratios and $95 \%$ confidence intervals for all-cause mortality by alcohol intake in Cox models controlling for predictors or potential confounders, but adding potential mediators stepwise

\begin{tabular}{lcccc}
\hline & Nil & Low & Moderate & Heavy \\
\hline Base model & 1.00 & $0.79(0.70-0.89)$ & $0.71(0.60-0.85)$ & $0.91(0.71-1.15)$ \\
Diabetes & 1.00 & $0.81(0.72-0.90)$ & $0.73(0.61-0.86)$ & $0.92(0.72-1.17)$ \\
Hypertension & 1.00 & $0.80(0.71-0.90)$ & $0.72(0.61-0.85)$ & $0.90(0.71-1.14)$ \\
Body Mass Index & 1.00 & $0.80(0.72-0.90)$ & $0.72(0.61-0.85)$ & $0.89(0.70-1.14)$ \\
HDL-Cholesterol & 1.00 & $0.80(0.72-0.90)$ & $0.73(0.61-0.87)$ & $0.91(0.71-1.16)$ \\
\hline
\end{tabular}

Table 4

Hazard ratios and 95\% confidence intervals for specified outcomes by alcohol intake in Cox models controlling for age, sex and other predictors or potential confounders

\begin{tabular}{lcccc}
\hline & Nil & Low & Moderate & Heavy \\
\hline CHD $(n=1088)$ & 1.00 & $0.86(0.75-0.99)$ & $0.99(0.81-1.22)$ & $0.65(0.46-0.93)$ \\
Ischaemic stroke $(n=420)$ & 1.00 & $0.77(0.61-0.98)$ & $0.93(0.67-1.29)$ & $0.83(0.49-1.40)$ \\
Dementia $(n=285)$ & 1.00 & $0.69(0.53-0.90)$ & $0.40(0.25-0.65)$ & $0.67(0.35-1.29)$ \\
\hline
\end{tabular}

CHD = coronary heart disease.

\section{Overall survival with alcohol and other risk factors}

We summed areas under survival curves for various predictors of ACM in the full Cox models. Over 20 years of follow-up, men and women taking any alcohol survived 12 months longer than their peers taking no alcohol. For smokers, men lost 41 months of survival, women 25 months; with hypertension, men lost 20 months, women lost 17 months; with diabetes, men lost 20 months, women lost 30 months; with atrial fibrillation, men lost 20 months, women lost 25 months.

\section{Alcohol, CVD and dementia}

Are the apparent protective effects of alcohol intake on ACM mediated through a relationship with cardiovascular disease in this elderly cohort? To increase statistical power we examined this question in relation to fatal and non-fatal coronary heart disease and ischaemic stroke events at 16 years follow-up. The findings are summarised in Table 4. There appears to be significant protection against cardiovascular disease in the low alcohol intake category, but the relationships appear inconsistent across the categories of intake.

Of particular interest in Table 4 is a significantly lower risk of onset of dementia at low and moderate alcohol intakes. This is demonstrated nicely in the "survival curves" free of dementia by alcohol intake category in the full Cox model shown in Fig. 5. Protection at heavy intake has very wide confidence intervals and may not be genuine.

\section{Discussion}

In this well-defined, community-based sample of elderly Australians, alcohol intake is best described as low to moderate. The results do confirm that intake of alcohol is associated with significantly reduced risk of ACM in both sexes in the familiar "U" shaped rela- 


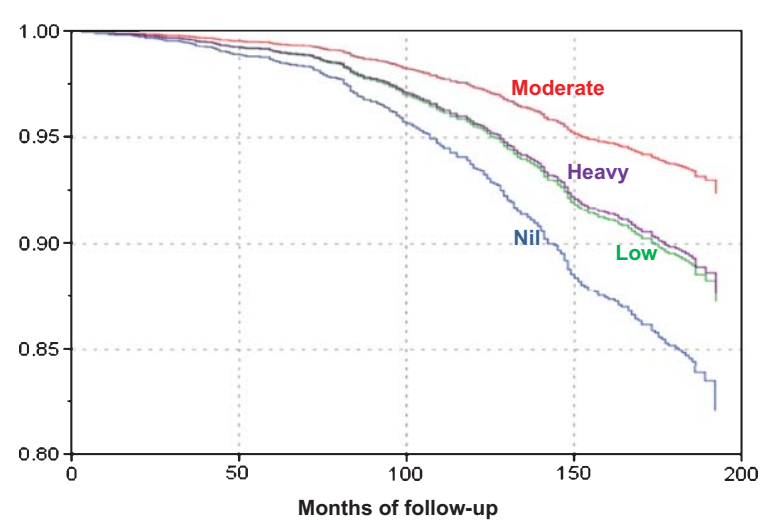

Fig. 5. Survival function free of dementia by category of alcohol intake in full Cox model.

tionship. Our findings have been broadly confirmed in other contemporary Australian studies [14, 15]. But this has not been a universal finding in all studies of the elderly, possibly because these cohorts may contain an excess of "healthy survivors", or there may be competing causes of mortality $[4,10,16,17]$.

There was a broadly similar relationship present with ACM whether subjects consumed predominantly beer or predominantly wine/spirits. It is unfortunate that we were unable to examine any separate effects of wine versus spirits. This study has other limitations which must be acknowledged. There is a potential for misclassification of alcohol intake between nil and low because of under-reporting. This would diminish any apparent relative benefit of alcohol intake since we have used nil intake as the reference group in the models. Hence, the findings may represent a minimum estimate of the benefit of low to moderate intake. Our categorisation of alcohol intake into low, moderate and heavy was arbitrary and based on sex-specific intake data, but this is a standard epidemiologic approach.

It is conceivable that factors such as diabetes, hypertension, body mass index or HDL cholesterol might have impacted or confounded the relationship of alcohol intake to ACM, both in a positive or negative sense. In particular, alcohol intake in Dubbo is positively correlated with HDL cholesterol in men $(r=0.32$, $P<0.001)$ and women $(r=0.23, P<0.001)$. However, the stepwise modelling in Table 3 showed no impact of any of these variables on the underlying relationship between alcohol intake and ACM. There was some protection by alcohol against cardiovascular disease, fatal or non-fatal, but relationships were inconsistent across intakes (Table 4). Alcohol may also influence survival in other ways which are currently not measured. It may reflect a special lifestyle integrated with less tangible factors [18].

While excess alcohol is undoubtedly toxic to the central nervous system, the finding of protection against dementia by alcohol intake in the low to moderate categories is interesting. But this outcome does find suggestive support from other studies [19-21] and it does merit further exploration in studies designed specifically for this purpose.

What are the public health implications of our findings, being derived from a population of "survivors" in their senior years? In the presence of low to moderate intake of alcohol, senior citizens can be encouraged to continue this behaviour.

\section{Conclusions}

1. Amongst Australian senior citizens in $1988,78 \%$ of men and $52 \%$ of women reported some alcohol intake. Most men reported an intake of 1-14 drinks/week, most women 1-7 drinks/week

2. $87 \%$ of male drinkers and $44 \%$ of female drinkers predominantly consumed beer. The remainder consumed wine and/or spirits

3. Over 20 years to $2008,64 \%$ of male drinkers died versus $72 \%$ of non-drinkers; $46 \%$ versus $60 \%$ in females

4. All-cause mortality was related to quantity of alcohol intake in the familiar "U" shaped relationship, being $20 \%$ and $28 \%$ reduced compared with nil intake in the low and moderate intake categories respectively. This relationship was similar with intake of beer or wine/spirits

5. Any alcohol intake versus none added 12 months survival time in men and women over 20 years follow-up

6. This relationship did not appear to be impacted or mediated by diabetes, hypertension, obesity or HDL cholesterol

7. Alcohol intake in the low to moderate range appears to offer protection against the onset of dementia

8. The overall Dubbo results are not unique but are applicable to a group of non-institutionalised senior citizens $60+$ years at study entry. A "healthy survivor" effect may be partially operating in this context 


\section{Acknowledgments}

The author acknowledges the major contributions to this study from co-investigators Professor John McCallum (Canberra), Professor Yechiel Friedlander (Jerusalem) and Mrs Judith Simons (Sydney).

\section{References}

[1] Klatsky AL, Armstrong MA, Friedman DD. Alcohol and mortality. Ann Intern Med 1992;(117):646-54.

[2] Holman CDJ, English DR, Milne E, Winter MG. Meta-analysis of alcohol and all-cause mortality: A validation of NHMRC recommendations. Med J Aust 1996;(164):142-5.

[3] Thun MJ, Peto R, Lopez AD, Monaco JH, Henley SJ, Heath CW, Doll R. Alcohol consumption and mortality among middle-aged and elderly US adults. N Engl J Med 1997;(337):1705-14.

[4] Di Castelnuovo A, Costanzo S, Bagnardi V, Donati MB, Iacoviello L, de Gaetano G. Alcohol dosing and total mortality in men and women. Arch Intern Med 2006;(166):2437-45.

[5] Rimm EB, Klatsky AL, Grobbee D, Stampfer MJ. Review of moderate alcohol consumption and reduced risk of coronary heart disease: Is the effect due to beer, wine, or spirits. BMJ 1996;(312):731-6.

[6] Di Castelnuovo A, Rotondo S, Costanzo S, Iacoviello L, Donati MB, de Gaetano G. Meta-analysis of wine and beer consumption in relation to vascular risk. Circulation 2002;(105):2836-44.

[7] Reynolds K, Lewis B, Nolen JD, Kinney GL, Sathya B, He J. Alcohol consumption and risk of stroke: A meta-analysis. JAMA 2003;(289):579-88.

[8] Gronbaek M, Deis A, Sorensen TIA, Becker U, Schnohr P, Jensen G. Mortality associated with moderate intakes of wine, beer or spirits. BMJ 1995;(310):1165-69.

[9] Renaud S, Geuguen R, Siest G, Salamon R. Wine, beer, and mortality in middle-aged men from Eastern France. Arch Intern Med 1999;(159):1865-70.

[10] Van de Water HA, Boshuizen HC. The impact of substitute morbidity and mortality on public health policies. Leiden: TNO
Prevention and Health, Division of Public health and Prevention. 1995

[11] Simons LA, McCallum J, Friedlander Y, Simons J, Powell I, Heller R. Dubbo Study of the elderly: Sociological and cardiovascular risk factors at entry. Aust N Z J Med 1991;(21): 701-9.

[12] Simons LA, McCallum J, Friedlander Y, Ortiz M, Simons J. Moderate alcohol intake is associated with survival in the elderly: The Dubbo Study Med J Aust 2000;(173):121-4.

[13] Simons LA, Simons J, Friedlander Y, McCallum J. Predictors of long-term mortality in the elderly: The Dubbo Study. Intern Med J 2011;(41):555-60.

[14] Cullen KJ, Knuiman MW, Ward NJ. Alcohol and mortality in Busselton, Western Australia. Am J Epidemiol 1993;(137):242-8.

[15] McCaul KA, Almeida OP, Hankey GJ, Jamrozik K, Byles JE, Flicker L. Alcohol use and mortality in older men and women. Addiction 2010;(105):1391-1400.

[16] Leino EV, Romelsjo A, Shoemaker C, Ager CR, Allebeck P, Ferrer HP, Fillmore, KM, Golding JM, Graves KL, Kniep S. Alcohol consumption and mortality. II. Studies of male populations. Addiction 1998;(93):205-18.

[17] Hart CL, Smith GD, Hole DJ, Hawthorne VM. Alcohol consumption and mortality from all causes, coronary heart disease, and stroke: Results from a prospective cohort study of Scottish men with 21 years follow up. BMJ 1999;(318):1725-9.

[18] Hommel M, Jaillard A. Alcohol for stroke prevention? N Engl J Med 1999;(341):1605-6.

[19] Orgogozo J-M, Dartigues J-F, Lafont S, Letenneur L, Commenges D, Salamon R, Renaud S, Breteler MB. Wine consumption and dementia in the elderly: A prospective community study in the Bordeaux area. Rev Neurol (Paris) 1997;(153):185-92.

[20] Peters R, Peters J, Warner J, Beckett N, Bulpitt C. Alcohol, dementia and cognitive decline in the elderly: A systematic review. Age Ageing 2008;(37):505-12.

[21] Kim JW, Lee DY, Jung MH, Kim H, Choi YS, Choi IG. Alcohol and cognition in the elderly: A review. Psychiatry Investig 2012;(9):8-16.

[22] National Heart Foundation of Australia and Australian Institute of Health. Risk Factor Prevalence Study: Survey No 3 1989, Cities Analysis. Canberra: NHFA and AIH, 1991. 